\title{
Dynamic interaction between the infinite beam and the the saturated poro-elastic soil foundation due to moving loads
}

\author{
HE Xi-ping \\ Evaluation Center of Education and Teaching, \\ Nanchang Institute of Technology, Jiangxi Nanchang \\ 330099, China \\ hxp@nit.edu.cn
}

XU Man-qing

School of Civil Engineering and Architecture, Nanchang Institute of Technology, Jiangxi Nanchang 330099, China

xmq418@163.com.cn

\author{
Huang Xiao-qiang \\ School of Civil Engineering and Architecture, \\ Nanchang Institute of Technology, Jiangxi Nanchang \\ 330099, China \\ HuangXQ@nit.edu.cn \\ Yang Zhao-ting \\ School of Civil Engineering and Architecture, \\ Nanchang Institute of Technology, Jiangxi Nanchang \\ 330099, China \\ Yangzt@nit.edu.cn
}

\begin{abstract}
For a saturated soil, a saturated poro-elastic model is more realistic than the linear elastic or the viscoelastic one. Based on the Fourier transform method in the frequency wave number domain, the expression of the equivalent stiffness of the saturated poro-elastic half space interacting with an infinite beam to harmonic moving loads is obtained. The time domain solutions for the beam and the saturated poro-elastic half space are derived by means of the inverse Fourier transform method. Also, the influences of the load speed, frequency and material parameters of the poroelastic half space on the responses of the beam are investigated. Numerical results show that the frequency corresponding to the maximum deflection and bending moment increases with increasing load speed. Also, the values of the maximum bending moment increases up to the critical speed. The load frequency corresponding to the maximum bending moment depends on the value of the load speed. With increasing load speed, spacing between frequencies increases.
\end{abstract}

Keywords- moving loads, saturated poro-elastic half space, Biot's theory, infinite beam, Fourier transform method

\section{INTRODUCTION}

Dynamic response of an infinite beam resting on a half space under moving loads has been a topic for engineering society for a long time, as the model can be used to simulate the railway subjected to moving train loads or various pavements subjected to moving vehicle loads. Majority of the papers addressing the dynamic response of an infinite beam on a half space to moving loads treat the half space as an elastic or a visco-elastic medium. For example, the steady-state vibration of a beam supported on an elastic half-space under a moving load has been studied in [1-5]. The dynamic response of beams on the generalized Pasternak visco-elastic foundations subjected to an arbitrary distributed harmonic moving load was analyzed in [6]. The response of an elastic beam on a visco-elastic layer to a uniformly moving constant load is investigated in $[7,8]$.

Recently some researchers have realized that high speeds trains will generate larger response for the rail and the ground especially for saturated soils, which may further cause noticeable structure-borne noise and vibration in the nearby buildings [9-11]. It is well known that the saturated soil is a two-phase material consisting of the soil skeleton and the pore water. Consequently, for a saturated soil, a saturated poro-elastic model is more realistic than the linear elastic or the visco-elastic one. Biot [12-14] pioneered the development of the theory for the saturated porous medium. Biot's theory has been widely applied in geomechanics to analyze consolidation effects due to quasi-static loads and wave propagation problems for dynamic loads.

\section{BIOT’S THEORY AND THE GENERAL SOLUTION}

The constitutive relations for the porous medium have the form [14]

$$
\begin{gathered}
\sigma_{i j}=\lambda \delta_{i j} \theta+2 \mu \varepsilon_{i j}-\alpha \delta_{i j} p, \\
p=-\alpha M \theta+M e, e=-w_{i, i}, \theta=u_{i, i}
\end{gathered}
$$

where $u_{i}$ and $w_{i}(i=1,2,3)$ are the displacement of solid skeleton and the infiltration displacement of the pore fluid, respectively; $\zeta_{i j}$ is the stress of the bulk material; $p$ is the pore pressure; $\varepsilon_{i j}$ and $\theta$ are the strain tensor and the dilatation of the solid skeleton, respectively; $e$ is the volume of fluid injected into a unit volume bulk material; $\delta_{i j}$ is the Kronecher delta; $\lambda$ and $\mu$ are Lame constants; $\alpha$ and $M$ are Biot's parameters accounting for compressibility of the porous medium.

The equations of motion for the bulk porous medium and the pore fluid have the form

$$
\begin{gathered}
\mu u_{i, j j}+\left(\lambda+\alpha^{2} M+\mu\right) u_{j, j i}-\alpha M w_{j, j i}=\rho+\rho_{\mathrm{f}} \\
\alpha M u_{j, j i}+M w_{j, j i}=\rho_{\mathrm{f}}+m+b_{p} \mathrm{~K}(t) * \alpha_{l}^{\&}
\end{gathered}
$$


where $\rho$ and $\rho_{f}$ are mass densities of the bulk material and the pore fluid, $\rho=(1-f) \rho_{s}+f \rho_{f} \cdot \rho_{s}$ is the density of the solid skeleton and $f$ is the porosity of the porous medium; $m=a_{\infty} \rho_{f} / f$ and $a_{\infty}$ is tortuosity; $b_{p}$ accounts for the viscosity of the pore fluid and the permeability of the porous medium, respectively and $\mathrm{K}(t)$ is a time-dependent viscosity correction factor which describes the transition behavior from viscosity dominated flow in the low frequency range towards inertia dominated flow at highfrequency rang [15]; the dot over a variable denotes the time derivative and the star $(*)$ between the two variables denotes the time convolution.

In order to solve Biot's governing equations, two kinds of Fourier transform are involved: the Fourier transform with respect to time and frequency and the Fourier transform with respect to horizontal coordinates and horizontal wave numbers. In this paper, the Fourier transform for time and the two horizontal coordinates are defined as follows

$$
\begin{aligned}
& \hat{f}(\omega)=\int_{-\infty}^{\infty} f(t) e^{-\mathrm{i} \omega t} d t, \quad f(t)=\frac{1}{2 \pi} \int_{-\infty}^{\infty} \hat{f}(\omega) e^{\mathrm{i} \omega t} d \omega \\
& \bar{f}(\xi)=\int_{-\infty}^{\infty} f(x) e^{-\mathrm{i} \xi x} d x, f(x)=\frac{1}{2 \pi} \int_{-\infty}^{\infty} \bar{f}(\xi) e^{\mathrm{i} \xi x} d \xi \\
& g /(\eta)=\int_{-\infty}^{\infty} f(y) e^{-\mathrm{i} \eta y} d y, f(y)=\frac{1}{2 \pi} \int_{-\infty}^{\infty} f^{g}(0 \eta) e^{\mathrm{i} \eta y} d \eta
\end{aligned}
$$

where the superimposed symbols $\wedge-\sim$ above a variable denote the Fourier transform with respect to time $t, x$ and $y$ coordinate, respectively.

Performing the Fourier transform with respect to time $t$ and Performing the double Fourier transform with respect to the two horizontal coordinates $x, y$ the dilatation of the solid, $\hat{\hat{u}} / 0$, stresses $\hat{\sigma}_{z z}^{0}$ is obtained as follows

$$
\begin{aligned}
i \xi \hat{u}_{x}^{0}= & v_{1}\left(A e^{\gamma_{1} z}+B e^{-\gamma_{1} z}\right)+v_{2}\left(C e^{\gamma_{2} z}+D e^{-\gamma_{2} z}\right) \\
& -\gamma_{3}\left(E e^{\gamma_{3} z}-F e^{-\gamma_{3} z}\right)-\mathrm{i} \eta\left(G e^{\gamma_{3} z}+H e^{-\gamma_{3} z}\right) \\
\hat{\hat{\sigma}}_{z z}^{0}= & \tau_{1}\left(A e^{\gamma_{1} z}+B e^{-\gamma_{1} z}\right)+\tau_{2}\left(C e^{\gamma_{2} z}\right. \\
+ & \left.D e^{-\gamma_{2} z}\right)+2 \mu \gamma_{3}\left(E e^{\gamma_{3} z}-F e^{-\gamma_{3} z}\right)
\end{aligned}
$$

where $\tau_{i}=\lambda\left(\chi_{j}-2 \mu a_{j} \gamma_{j}^{2}\right),(j=1,2)$

\section{THE MODEL FOR A BEAM RESTING ON A SATURATED PORO-ELASTIC HALF SPACE SUBJECTED TO A HARMONIC MOVING LOAD}

Fig .1 illustrates an infinite Euler-Bernoulli beam resting on a saturated poro-elastic half space and subjected to a harmonic line moving load with a constant velocity. For simplicity, the following assumptions are made for the beam and the load: (a) the beam is treated as an infinite Euler-Bernoulli elastic beam with a width $2 a$; (b) the deformation of the beam is infinitesimal; (c) the shear deformation and the rotary inertia of the beam are negligible; (d) both the moving load and the normal stresses between the beam and the half space are uniformly distributed over the width of the beam; (e) the contact between the beam and the half space is smooth.
According to the elastic beam theory, the equation of motion for the beam is as follows

$$
E I_{z} \frac{\partial^{4} w_{b}(x, t)}{\partial^{4} x}+m_{b} \frac{\partial^{2} w_{b}(x, t)}{\partial^{2} t}=F(x, t)-q_{z}(x, t)
$$

where $w_{b}(x, t)$ is the deflection of the beam, $E$ Young's modulus of beam material, $I_{z}$ the second moment of area of the beam cross section about its neutral axis (Fig .1), $q_{z}(x, t)$ is the interaction force between the beam and the half space, $F(x, t)$ is the applied moving load which is uniformly distributed over the width. The moving load $F(x, t)$ is a line load moving with a constant velocity $c$ and given by the following expression

$$
F(x, y, t)=F_{\mathrm{z}} \delta(x-c t) e^{\mathrm{i} \omega_{0} t}
$$

where $\omega_{0}, F_{z}$ is the original frequency, the magnitude of the moving load, respectively, $\delta(\cdots)$ is the Dirac delta function.

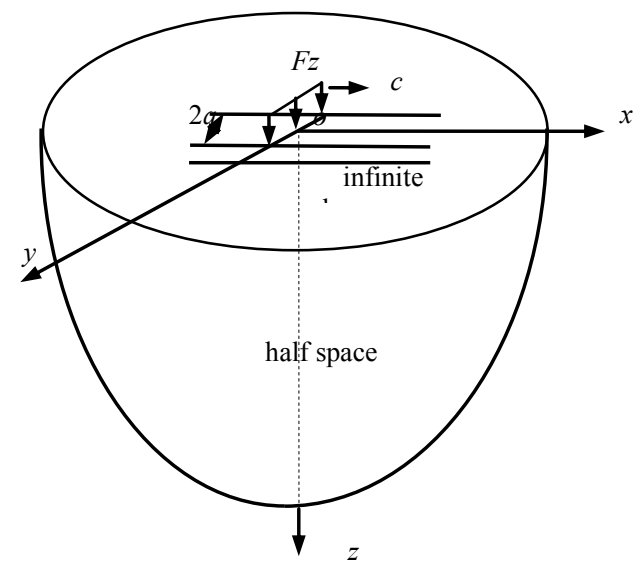

Figure 1. Model of an infinite beam overlying a layered poro-elastic half space subjected to moving loads

According to the assumptions concerning the beam, the stress boundary conditions for the surface of the saturated half space are as follows

$$
\begin{gathered}
2 a \sigma_{z z}(x, y, 0, t)=q_{z}(x, t) H(a-y) H(a+y) \\
\sigma_{x z}(x, y, 0, t)=0, \sigma_{y z}(x, y, 0, t)=0
\end{gathered}
$$

where $H(\cdots)$ is the unit step function. Moreover, the following "'open pore" boundary conditions is assumed for the surface of the poro-elastic half space [16]

$$
p(x, y, 0, t)=0
$$

Applying the two-dimensional inverse Fourier transformation for $\xi \rightarrow x$ and $\omega \rightarrow t$, the expressions for the bending moment and the shear force of the beam have the following form

$$
\begin{aligned}
& M_{x}(x, t)=-\frac{F_{z}}{2 \pi} \int_{-\infty}^{\infty} \frac{E I_{z} \xi^{2} e^{i \xi(x-c t)} e^{i \omega_{0} t}}{E I \xi^{4}-m_{b}\left(\omega_{0}-\xi c\right)^{2}+k_{e q}\left(\xi, \omega_{0}-\xi c\right)} d \xi \\
& Q_{x}(x, t)=-\frac{F_{z}}{2 \pi} \int_{-\infty}^{\infty} \frac{E I_{z} \xi^{3} e^{i \xi(x-c t)} e^{i \omega_{0} t}}{E I_{z} \xi^{4}-m_{b}\left(\omega_{0}-\xi c\right)^{2}+k_{e q}\left(\xi, \omega_{0}-\xi c\right)} d \xi
\end{aligned}
$$

\section{NUMERICAL RESULTS}

In this section, the half space consists of a saturated poro-elastic foundation with depth $h$, and the bottom 
foundation is a rigid and impermeable bedrock. The line load moves along the positive $x$-axis with a constant velocity $c$ and the load is normal to the beam. In calculation, the parameters for the beam and the saturated foundation are as follows: $E I_{z}=1.3 \times 10^{9} \mathrm{~N} \cdot \mathrm{m}^{2}$, $m_{b}=1770 \mathrm{~kg} / \mathrm{m} \quad, \quad a=1.3 \mathrm{~m}, \mu=3.8 \times 10^{7} \mathrm{~N} / \mathrm{m}^{2}$, $\lambda=3.8 \times 10^{7} \mathrm{~N} / \mathrm{m}^{2} \quad, \quad M=2.4 \times 10^{8} \mathrm{~N} / \mathrm{m}^{2}$ ， $\rho_{s}=2.0 \times 10^{3} \mathrm{~kg} / \mathrm{m}^{3}, \rho_{f}=1.0 \times 10^{3} \mathrm{~kg} / \mathrm{m}^{3}, f=0.35$, $\alpha=0.97, b_{p}=1.94 \times 10^{6} \mathrm{~kg} / \mathrm{m}^{3} \mathrm{~s}, \quad m=1990 \mathrm{~kg} / \mathrm{m}^{3}$, $\omega_{0}=0$. The reference shear wave velocity is defined as $v_{\mathrm{SH}}=\sqrt{\mu / \rho_{s}}$. In this section, the equivalent stiffness of the system is calculated at first. Then, the influences of the depth of the saturated poro-elastic foundation on the vertical vibration and the internal forces of the beam are discussed.

In this section, the vertical displacement, the bending moment, the shear force of the beam and the foundation depth $h$ are normalized as follows: $u^{*}=\mu_{R} w_{b} / F_{z}$, $M^{*}=M_{x} /\left(F_{z} a_{R}^{2}\right), Q^{*}=Q_{x} /\left(F_{z} a_{R}^{2}\right), h^{*}=h / a_{R}$. The reference shear modulus and the reference length are: $\mu_{R}=3.8 \times 10^{7} \mathrm{~N} / \mathrm{m}^{2}, a_{R}=a$, respectively.

The maximum vertical deflection of the beam $u^{*}$ versus the moving load velocity $c$ is shown in Fig .2 for different depth $h^{*}$. Fig .2 indicates that at first the maximum deflection of the beam increases as the depth of the saturated foundation increases. However, when the depth reaches $h^{*}=20.0$, the increment is difficult to perceive.

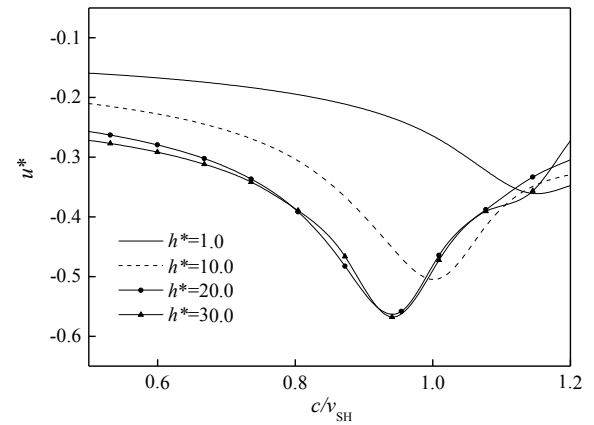

Figure 2. Maximum vertical deflection of the beam versus moving load velocity for different depths of the overlying layer

Fig .3 (a) and 5 (b) illustrates the bending moment and the shear force for the infinite beam at the observation point $\mathrm{P}(0.0 \mathrm{~m}, 0.0 \mathrm{~m}, 0.0 \mathrm{~m})$ versus time $t$ for two depths of the saturated foundation: $h^{*}=1.0$ and $h^{*}=20.0$. The velocity of the moving load is $c=0.7 v_{\mathrm{SH}}$ Time $t=0.0 \mathrm{~s}$ corresponds to the instant at which the applied load is passing through the origin. Fig .5 shows that the increasing depth of the layer enhances the bending moment and the shear force of the beam.

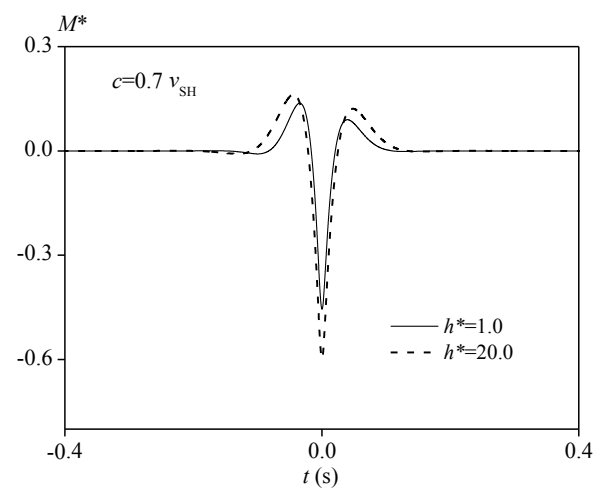

(a)

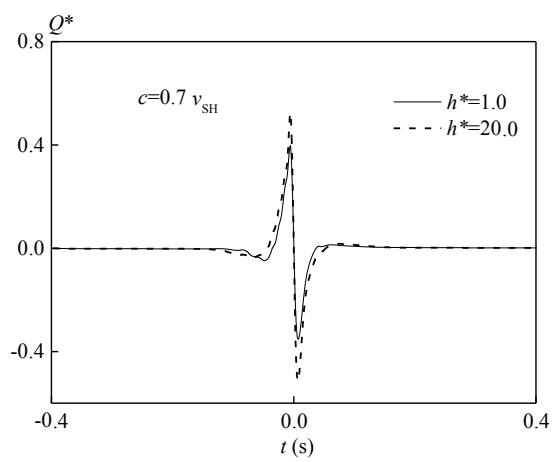

(b)

Figure 3. Internal force for the infinite beam subjected to moving load with a constant velocity $c=0.7 v_{\mathrm{SH}}$ at the observation point P $(0.0 \mathrm{~m}, 0.0 \mathrm{~m})$ layer, $h^{*}=1.0$ and $h^{*}=20.0$ : (a) the bending moment versus time $\mathrm{t}$; (b) the shear force versus time $\mathrm{t}$

The effect of load frequency and speed on the maximum deflection is illustrated in Fig. 4. It follows from Fig .6 for speeds less than critical speed $\left(c \approx v_{\mathrm{SH}}\right)$, the maximum deflection increases with increasing load frequency and after reaching a maximum value it decreases with increasing frequency. The frequency corresponding to the maximum deflection depends on the load speed. For the case with a load speed equal to the critical speed, the maximum deflection decreases with the increasing load frequency. For the cases with speeds larger than the critical speed, the maximum deflection increases first and then it decreases.

The effect of load frequency and speed on the maximum bending moment is illustrated in Fig .5. For speeds less than critical speed $\left(c \approx v_{\mathrm{SH}}\right)$, the maximum bending moment increases with increasing load frequency; however, after reaching to a maximum value it decreases. Also, the values of the maximum bending moment increases up to the critical speed. The load frequency corresponding to the maximum bending moment $\left(\omega_{\mathrm{p}}\right)$ depends on the value of the load speed. With increasing load speed, spacing between frequencies $\left(\omega_{\mathrm{p}}\right)$ increases. For the case with the load speed equal to the critical value, 
the maximum bending moment decreases within the calculation range. For the cases $\left(c>v_{\mathrm{SH}}\right)$, the maximum bending moment increases first (up to $c=2.5 v_{\mathrm{SH}}$ ) and then decreases.

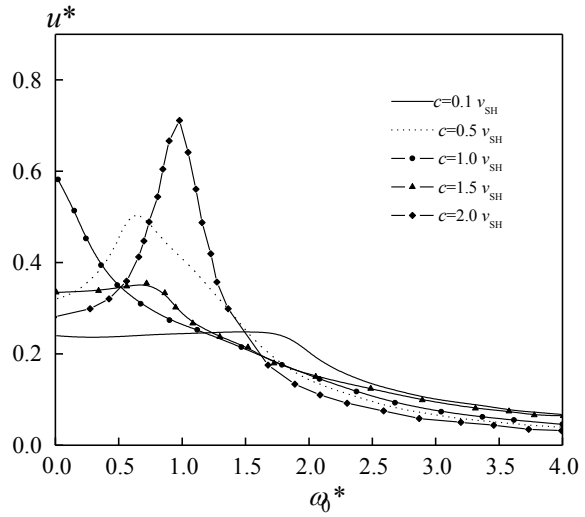

Figure 4. Velocity and frequency of moving load effect on the maximum of deflection of the beam $u^{*}$

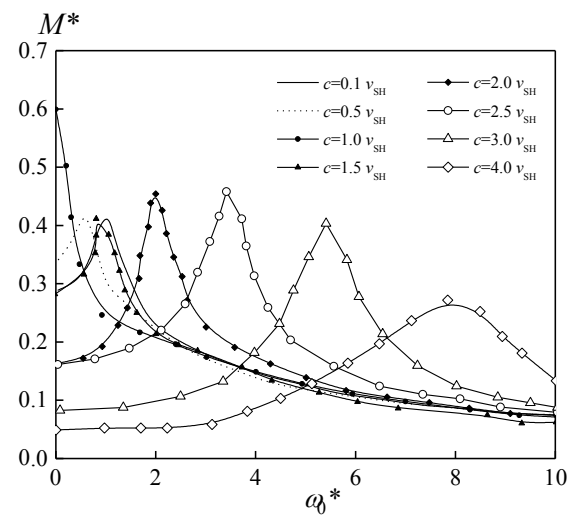

Figure 5. Velocity and frequency of moving load effect on the maximum of bending moment of the beam $M *$

\section{CONCLUSION}

Dynamic response of an infinite beam resting on a saturated poro-elastic half space subjected to harmonic moving loads is addressed in this study. The equivalent stiffness of the saturated porous half space is derived by means of the Fourier transform method. Based on the proposed methodology, the deflection, the bending moment and the shear force of the beam are obtained. Response of the half space due to the moving load is also calculated. The influences of the load speed, frequency and parameters of the poro-elastic half space on the beam responses are investigated. It follows from the numerical results that the frequency corresponding to the maximum deflection and bending moment increases with increasing load speed.

\section{ACKNOWLEDGMENT}

The project is supported by the Jiangxi Provincial Natural Science Fund Project(NO: 20132BAB206004),
Scientific Research Fund of Jiangxi Provincial Education Department(NO: GJJ14755) and the College Students' Innovation and Entrepreneurship Education Special Funds as well as the Nanchang Institute of Technology Scientific Research Training Plan for the College Students in 2013.

\section{REFERENCES}

[1] Metrikine AV, Dieterman HA. Three-dimensional vibrations of a beam on and elastic half-space: resonance interaction of vertical longitudinal and lateral beam waves. Journal of Applied Mathematics and Mechanics 1997; 64: 951-956.

[2] Lipen AB, Chigarev AV. The displacements in an elastic halfspace when a load moves along a beam lying on its surface. Journal of Applied Mathematics and Mechanics 1998; 62 (5): 791-796.

[3] Sheng X, Jones CJC, Petyt M. Ground vibration generated by a harmonic load acting on a railway track. Journal of Sound and Vibration 1999; 225: 3-28.

[4] Madshus C, Kaynia AM. High-speed railway lines on soft ground: dynamic behavior at critical train speed. Journal of Sound and Vibration 2000; 231: 689-701.

[5] Takemiya H. Simulation of track-ground vibrations due to a highspeed train: the case of X-2000 at Ledsgard. Journal of Sound and Vibration 2003; 261: 503-526.

[6] Kargarnovin MH, Younesian D. Dynamics of Timoshenko beams on Pasternak foundation under moving load. Mechanics Research Communications 2004; 31:713-723.

[7] Metrikine AV, Popp K. Steady-state response of an elastic beam on a visco-elastic layer under moving load. Archive of Applied Mechanics 2000; 70: 399-408.

[8] Vostroukhov AV, Metrikine AV. Periodically supported beam on a visco-elastic layer as a model for dynamic analysis of a high-speed railway track. International Journal of Solids and Structures 2003; 40: 5723-5752.

[9] Auersch L. The excitation of ground vibration by rail traffic: theory of vehicle-track-soil interaction and measurements on high-speed lines. Journal of Sound and Vibration 2005; 284: 103-132.

[10] Auersch L. Dynamics of the railway track and the underlying soil: the boundary-element solution, theoretical results and their experimental verification. Vehicle System Dynamics 2005; 43 671-695.

[11] Jin B. Dynamic displacement of an infinite beam on a poroelastic half space due to a moving oscillating load. Archive of Applied Mechanics 2004; 74: 277-287.

[12] Biot MA. Theory of propagation of elastic waves in a fluidsaturated porous solid, I, Low frequency range. Journal of the Acoustical Society of America 1956; 28: 168-178.

[13] Biot MA. Theory of propagation of elastic waves in a fluidsaturated porous solid, II: Higher frequency range. Journal of the Acoustical Society of America 1956; 28: 179-191.

[14] Biot MA. Mechanics of deformation and acoustic propagation in porous media. Journal of Applied Psychology 1962; 33: 14821498.

[15] Johnson DL, Koplik J, Dashen R. Theory of dynamic permeability and tortuosity in fluid-saturated porous-media. Journal of Fluid Mechanics 1987; 176; 379-402.

[16] Deresiewicz H, Skalak R. On the uniqueness in dynamic poroelasticity. Bulletin of the Seismological Society of America $1963 ; 53: 783-788$.

[17] Oppenheim AV, Schafer RW. Discrete-time signal processing. 1999; Prentice-Hall, Inc., Englewood Cliffs, NJ. 